\title{
La Investigación en el cultivo de papa en la Universidad Pedagógica y Tecnológica de Colombia
}

\section{Research on potato crop at the Universidad Pedagógica y Tecnológica de Colombia}

\section{Pesquisa em cultivo de batata na Universidade Pedagógica e Tecnológica da Colômbia}

\author{
Lizeth Johana Sierra-Ariza ${ }^{1}$, Katherin Dayana Suárez-Roberto ${ }^{2}$, Jorge Enrique Villamil-Carvajal ${ }^{3}$ y \\ Álvaro Enrique Alvarado-Gaona ${ }^{4}$.
}

\section{Recibido:}

Febrero 15 de 2017

Aceptado:

Junio 7 de 2017

${ }^{1}$ Estudiante de Ingeniería Agronómica.

lizjoha34@hotmail.com. Orcid: 0000-0002-5441-9152.

Universidad Pedagógica y

Tecnológica de Colombia. Tunja-Colombia.

${ }^{2}$ Estudiante de Ingeniería Agronómica, katehafneo@live.

com.

Orcid: 0000-0003-2772-0924

Universidad Pedagógica y

Tecnológica de Colombia. Tunja-Colombia.

${ }^{3}$ Ingeniero Agronómico. jvillamil@corpoica.org.co. Orcid: 0000-0003-0173-0921 Corporación Colombiana de Investigación Agropecuaria. Tunja-Colombia.

${ }^{4}$ Magíster en Desarrollo Rural alvaro.alvarado@uptc.edu.co. Orcid: 0000-0002-7024-5594

Universidad Pedagógica y Tecnológica de Colombia. Tunja-Colombia

\section{Resumen}

Antecedentes: La papa (Solanum tuberosum L. grupo Andigena y Phureja) es un tubérculo de vital importancia en Colombia y en especial en el departamento de Boyacá. En la Universidad Pedagógica y Tecnológica de Colombia (UPTC) se cuenta con más de mil trabajos de grado, realizados en los 55 años de trayectoria del programa académico de Ingeniería Agronómica, entre los cuales 130 corresponden a investigaciones relacionadas con este cultivo. Objetivo: se recopiló información de dichos trabajos de grado desarrollados en este período y se analizaron 5 áreas de investigación: entomología, nutrición, fisiología vegetal, fitopatología y economía, en 3 lapsos de tiempo de 10 años cada uno y un último de 12 años. Resultados: En entomología se adelantaron 42 trabajos de grado (32,3\%), siendo los de gusano blanco (Premnotrypes vorax Hustache) los más representativos. En el área de nutrición con 35 trabajos de grado $(26,9 \%)$, se resaltaron los temas sobre correctivos de acidez y nutrición con elementos mayores N-P-K, haciendo énfasis en la evaluación del uso del fósforo. En fisiología vegetal se documentaron 20 trabajos de grado $(15,4 \%)$, dentro de los cuales, se destacaron los temas sobre estadios fenológicos, crecimiento y desarrollo del cultivo de papa. En economía con 18 trabajos de grado $(13,8 \%)$, fue más importante la evaluación económica y respuesta agronómica en fertilización, y finalmente en fitopatología con 15 trabajos de grado (11,5\%), el tizón tardío (Phytophthora infestans) correspondió al patógeno más estudiado. Conclusión: Esta revisión brinda información a estudiantes, profesionales e instituciones involucradas con el sector papero colombiano y departamental, para la orientación y enfoque de futuros trabajos de investigación.

Palabras clave: Economía, Entomología, Fisiología vegetal, Fitopatología, Nutrición.

\begin{abstract}
Background: Potato (Solanum tuberosum L. Andigena y Phureja groups) is a very important tuber in Colombia, mainly in the Boyacá department. At the Universidad Pedagógica y Tecnológica de Colombia (UPTC) students had developed over one thousand thesis, along 55 years of the agronomic engineer academic program, 130 of those related with
\end{abstract}


potato crop. Objective: Information provided by those thesis during that period was taken together, and five research lines were analyzed: entomology, fertilization, plant physiology, phytopathology and economy in three timeframes of ten years and a last one of twelve years. Results: In the entomology area 42 studies were performed (32.3\%), being Andean potato weevil (Premnotrypes vorax Hustache) the most representative. In the nutrition area with 35 studies $(26.9 \%)$, the topics on soil acidity management were highlighted and plant nutrition with N-P-K elements, emphasizing in phosphorous use. In plant physiology 20 studies (15.4\%) were documented, among which, phenology and potato growth and development were the most relevant. In the economic area with 18 studies (13.8\%), the most important topic was economic evaluation and agronomic response to fertilization; and finally, in phytopathology with 15 studies $(11.5 \%)$, late blight (Phytophthora infestans) was the most studied pathogen. Conclusion: This review brings information to students, professionals and institutions involved in potato crop production in Colombia and Boyacá, giving guidelines and focus for future potato research.

Keywords: economy, entomology, nutrition, plant physiology, phytopathology.

\section{Resumo}

Antecedentes: A batata (Grupo Solanum tuberosum L. Andigena e Phureja) é um tubérculo de vital importância na Colômbia e especialmente no departamento de Boyacá. Na Universidade de Pedagogia e Universidade Tecnológica da Colômbia (UPTC) existem mais de mil estudos de graduação, realizados nos 55 anos do programa acadêmico de Engenharia Agrícola, dentre os quais 130 correspondem a pesquisas relacionadas a esta safra. Objetivo: compilamos informações sobre esses estudos de pós-graduação desenvolvidos neste período e analisamos 5 áreas de pesquisa: entomologia, nutrição, fisiologia vegetal, fitopatologia e economia, em 3 períodos de 10 anos cada e nos últimos 12 anos. Resultados: na entomologia, 42 estudos de graduação $(32,3 \%)$ foram avançados, com o verme branco (Premnotrypes vorax Hustache) sendo o mais representativo. $\mathrm{Na}$ área de nutrição com empregos de 35 graus (26,9\%), foram enfatizados os sujeitos sobre a acidez e corretivos nutricionais com elementos N-P-K mais altos, com ênfase na avaliação do uso de fósforo. Vinte estudos de pós-graduação $(15,4 \%)$ foram documentados em fisiologia vegetal, com ênfase em estádios fenológicos, crescimento e desenvolvimento de cultivo de batata. 18 postos de trabalho no grau da economia (13,8\%), avaliação económica e resposta à fertilização Agronômico era mais importante, e, finalmente, em Fitopatologia 15 obras de graduação $(11,5 \%)$, requeima (Phytophthora infestans) correspondeu para o patógeno mais estudado. Conclusão: esta revisão fornece informações aos alunos, profissionais e instituições envolvidas com o setor de papelaria colombiano e departamental, para orientar e focar futuros trabalhos de pesquisa.

Palavras-chave: economia, entomologia, nutrição, fisiologia vegetal, fitopatologia.

\section{Introducción}

La papa (Solanum tuberosum L.) constituye el cuarto cultivo alimenticio de mayor consumo en el mundo [1]. En el 2013 la producción a nivel mundial fue de $368.096 .362 \mathrm{t}$, y para Colombia $2.788 .050 \mathrm{t}$, siendo los principales departamentos productores Cundinamarca con 1.001.376 t y Boyacá con 709.000 t [2]. $S$. tuberosum es una herbácea de la cual el tubérculo es la parte comercial donde este además de almacenar nutrientes, tiene alto contenido de carbohidratos y en menor medida proteínas, fibra, calcio, hierro y vitamina C [3]. La planta tiene hojas compuestas, flores color blanco, púrpura o veteadas, según la 
No. 2

Julio - Dic. 2017

ISSN 0122-820X

E-ISSN 2422-5053

PP: $102-115$ variedad; el fruto, es redondo con un diámetro de $2 \mathrm{~cm}$ aproximadamente [4]. Las principales enfermedades en este cultivo son: el tizón tardío (Phytophthora infestans (Mont.) de Bary), la pata negra (Erwinia carotovora var. atroseptica (van Hall) Dye) y antracnosis (Colletotrichum gloeosporioides (Penz.) Sacc.). Las plagas más limitantes son: polilla guatemalteca (Tecia solanivora Povolny (Lepidóptera: Gelechiidae)), gusano blanco (Premnotrypes vorax Hustache (Coleoptera: Cuculionidae), nemátodos (Globodera spp., Nacobbus averrans) y áfidos (Macrosiphum euphorbiae Thomas y Myzus persicae Sulzer (Hemiptera: Aphididae)) [5].

La presente revisión tuvo como objetivo recopilar información de los trabajos de grado realizados durante 55 años sobre el cultivo de la papa (S. tuberosum) por el programa de Ingeniería Agronómica de la Universidad Pedagógica y Tecnológica de Colombia (Tunja, Colombia), analizando 5 áreas de investigación: insectos-plaga, fertilización, fisiología vegetal, economía y fitopatología; en aras de aportar elementos que brinden información a estudiantes, profesionales e instituciones involucradas con el sector papero colombiano y departamental, para la orientación y enfoque de futuros trabajos de investigación.

\section{Materiales y métodos}

El presente estudio se realizó en la Universidad Pedagógica y Tecnológica de 104 Colombia (UPTC) sede principal-Tunja, en las instalaciones de la biblioteca Jorge Palacios Preciado, área de colecciones especiales sala de trabajos de grado, durante el segundo semestre de 2015. Este trabajo fue de tipo descriptivo; el cual, involucró la caracterizaron de las tesis de grado desde 1967-2012 (45 años) de la escuela de Ingeniería Agronómica en el cultivo de papá en cinco áreas claves del conocimiento: entomología, fitopatología, fisiología, nutrición y economía. Se determinaron las siguientes variables: número de trabajos de grado por área publicados anual y quinquenalmente, número de integrantes $\mathrm{y}$ género por trabajo de grado, nombre de director y género, área y tema específico. Los resultados son mostrados mediante análisis descriptivos por medio de gráficos de líneas, barras verticales, barras apiladas y diagramas de Pareto.

\subsection{Indicadores bibliométricos}

Indicadores de productividad personal: número y género de estudiantes y directores que ejecutaron el trabajo, índice de productividad por director cada cinco años, y por área específica: definido como el logaritmo decimal del número de trabajos de grado realizados [6]. Por tanto si $I P \geq 1$ indica la producción de 10 trabajos de grado o más debido a que el $\log 10$ es 1 ; sin embargo sí $I P=0$ indica la producción de un solo trabajo, porque el log de 1 es 0 [7], [8].

\section{Resultados y análisis}

Durante la trayectoria de los 55 años del programa de Ingeniería Agronómica, se desarrollaron 130 trabajos de grado en las áreas de: entomología, la cual evidenció el mayor número de trabajos con 42 , equivalente al $32,3 \%$, seguida por la de nutrición con 35 (26,9\%), fisiología $20(15,4 \%)$ economía 18 $(13,8 \%)$ y fitopatología $15(11,5 \%)$ con la menor cantidad de estudios. Al relacionar el índice de productividad (IP), el mayor valor se registró con los estudios de entomología $(1,6)$, seguidos por los de nutrición $(1,5)$ (Gráfica 1). 


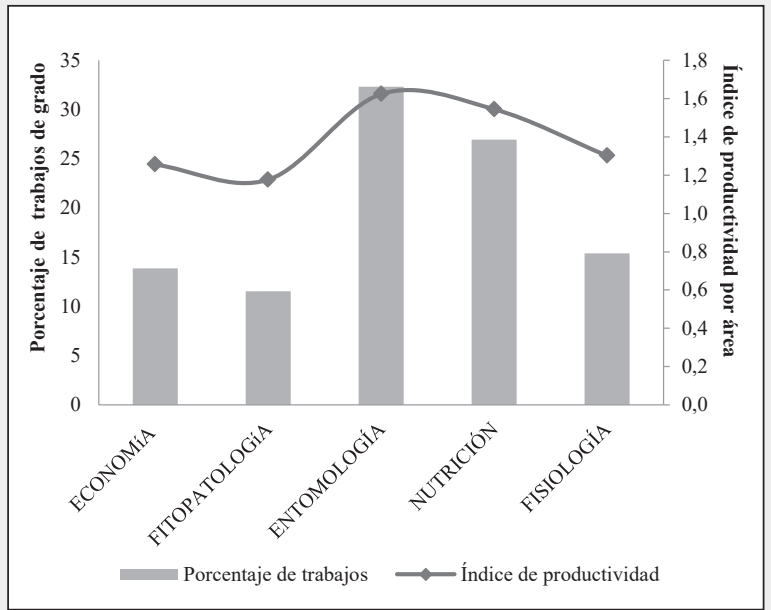

Gráfica 1. Trabajos de grado e índices de productividad (IP) en el cultivo de papa para 5 áreas de estudio durante el periodo 1967 a 2012. Escuela Ingeniería Agronómica, Universidad Pedagógica y Tecnológica de Colombia-UPTC, Tunja-Boyacá, Colombia.

La media general de trabajos de grado publicados por año para las cinco áreas en estudio fue de $2,83 \pm 0,35$, y a nivel individual, entomología presentó el mayor número promedio de trabajos con $0,91 \pm 0,22$, seguido en su orden por nutrición $(0,76 \pm 0,16)$, fisiología $(0,43 \pm 0,22)$, economía $(0,39 \pm 0,09)$, y fitopatología $(0,33 \pm 0,08)$ con la menor cantidad de tesis. La gráfica 2, muestra el número total de trabajos publicados durante cinco años por cada área. Para 1967 a 1970 (3 primeros años), se observó el menor índice de productividad -IP $(0,67)$, siendo entomología la línea más estudiada (4 tesis). Para los años 1991 a 1995 se destacaron los trabajos en nutrición (12 estudios), y para el periodo 1996 a 2000 en entomología (19 trabajos), siendo este el quinquenio con el mayor índice de productividad $(1,47)$.

Durante los últimos años (2001-2005, 20062012) el enfoque de investigación fue en el área de fitopatología (4 tesis) y fisiología (13 tesis) respectivamente.

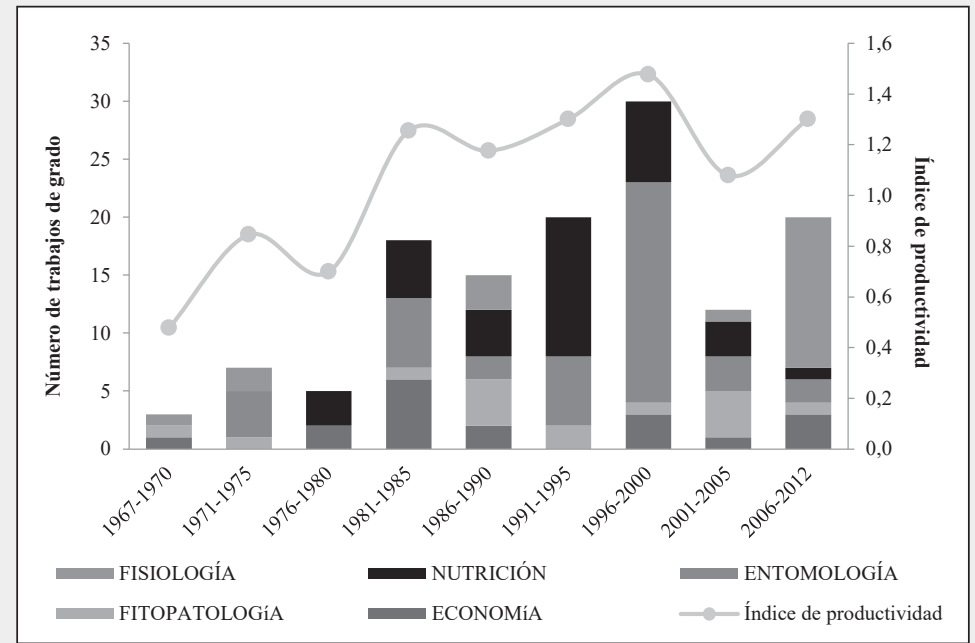

Gráfica 2. Trabajos de grado e índices de productividad, publicados por quinquenio en el cultivo de papa para 5 áreas de estudio durante el periodo 1967 a 2012. Escuela Ingeniería Agronómica, UPTC, Tunja-Boyacá, Colombia.

En el periodo de 1967 a 1980 el promedio de trabajos de grado publicados fue de $1,1 \pm 0,27$, y a partir de 1981 existió una tendencia de aumento en el número de tesis hasta el año $2000(4,2 \pm 1,07)$. Para los últimos 12 años, hubo nuevamente un descenso en productividad $(2,6 \pm 0,79)$, lo cual, está relacionado con las diversas opciones de grado que tiene la universidad, donde los estudiantes ya no prefieren hacer tesis como requisito de graduación (Gráfica 3). 


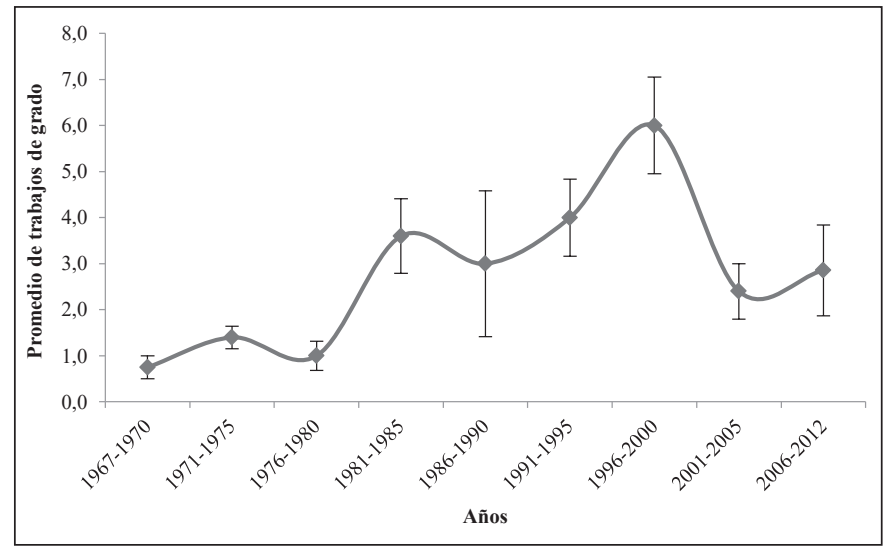

Gráfica 3. Promedio general de los trabajos de grado publicados por quinquenio en el cultivo de papa para 5 áreas de estudio, durante 1967 a 2012. Escuela Ingeniería Agronómica, UPTC, Tunja-Boyacá, Colombia. Los intervalos sobre la curva representan el error estándar.

Tabla I. Relación del género de estudiantes tesistas. Escuela Ingeniería Agronómica, UPTC, Tunja-Boyacá, Colombia.

\begin{tabular}{|c|c|c|c|c|}
\hline ÁREA & $\begin{array}{c}\text { NÚMERO } \\
\text { ESTUDIANTES }\end{array}$ & $\begin{array}{c}\text { PORCENTAJE } \\
\text { ESTUDIANTES (\%) }\end{array}$ & $\begin{array}{c}\text { PORCENTAJE } \\
\text { MUJERES (\%) }\end{array}$ & $\begin{array}{c}\text { PORCENTAJE } \\
\text { HOMBRES (\%) }\end{array}$ \\
\hline Economía & 29 & 13,4 & 31,0 & 69 \\
\hline Fitopatología & 23 & 10,6 & 13 & 87 \\
\hline Entomología & 76 & 35,2 & 25 & 75 \\
\hline Nutrición & 61 & 28,2 & 11,5 & 88,5 \\
\hline Fisiología & 27 & 12,5 & 22,2 & 77,8 \\
\hline General & 216 & 100 & 20,4 & 79,6 \\
\hline
\end{tabular}

Los trabajos de grado fueron desarrollados por 216 estudiantes, de los cuales el $79,6 \%$ correspondió a hombres y $20,4 \%$ a mujeres. Se destacó la participación femenina $(31 \%)$ en el área de economía y la masculina $(88,5 \%)$ en nutrición del cultivo (Tabla I).

En total se registraron 44 directores de trabajos de grado para el periodo 1967 a 2012. La gráfica 4, muestra los 20 directores que

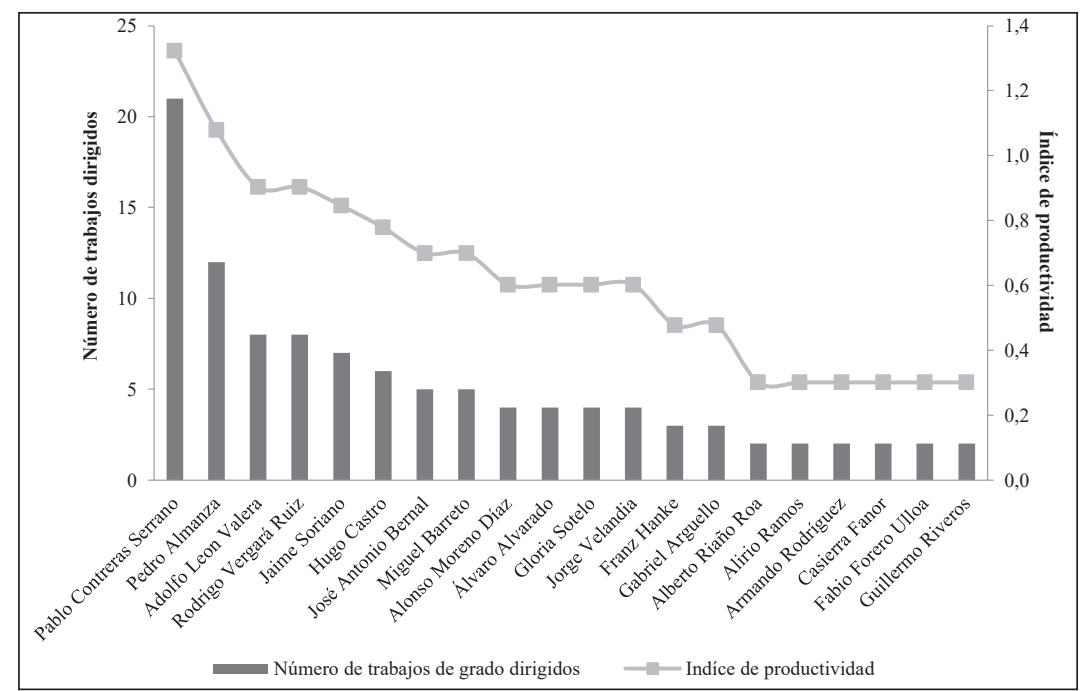

Gráfica 4. Relación de directores e índices de productividad, con el mayor número de trabajos de grado publicados en el cultivo de papa para 5 áreas de estudio, durante 1967 a 2012 . Escuela Ingeniería Agronómica, UPTC, Tunja-Boyacá, Colombia. 
Lizeth Johana Sierra-Ariza, Katherin Dayana Suárez-Roberto, Jorge Enrique Villamil-Carvajal, Álvaro Enrique Alvarado-Gaona

sobresalieron por la orientación de 2 o más tesis de grado, donde se destacó el profesor Pablo Contreras Serrano con el mayor índice de productividad $(1,3)$ seguido por Pedro José Almanza (1,07).

\subsection{Entomología}

El periodo transcurrido desde 1989 a 1999, fue la época en la cual se desarrolló el principal número de trabajos de grado con 23 proyectos (54,7\%), seguido por los años comprendidos entre 1978-1988 con 8 (19\%), 2000-2012, 7 $(16,6 \%)$ y 1967 a $1977,4(9,5 \%)$ con la menor cantidad de investigaciones. En las diferentes tesis de grado adelantadas por estudiantes del programa de Ingeniería Agronómica, $P$. vorax ha sido el insecto-plaga más estudiado [9], el cual, se considera plaga clave en la producción desde hace más de 90 años, al ocasionar galerías dentro del tubérculo que afectan la calidad de la cosecha y causan pérdida total del cultivo cuando el ataque es severo [10], [11] en consecuencia puede existir abandono del mismo [12], generando pérdidas superiores a 800 millones de pesos, como sucedió en 1980 [13]. En cuanto al manejo de este coleóptero, el control químico fue el más evaluado por ser la práctica empleada por el productor, quien, en la mayoría de casos aplica insecticidas sin tener en cuenta umbrales de daño, lo que ha generado resistencia del insecto a ciertos grupos químicos de uso convencional [11]. Resultados similares fueron observados por [14], al encontrar eficiencia del 93,4\% con el uso de Carbofuran tres veces en la zona periférica de la parcela de papa a manera de barrera química.

El estudio sobre control [15] biológico y cultural como estrategia del manejo integrado de plagas (MIP), se reporta a partir de 1995 [16] con la evaluación de hongos entomopatógenos en almacenamiento y en campo sobre $P$. vorax, siendo esta medida de control la segunda práctica más estudiada.

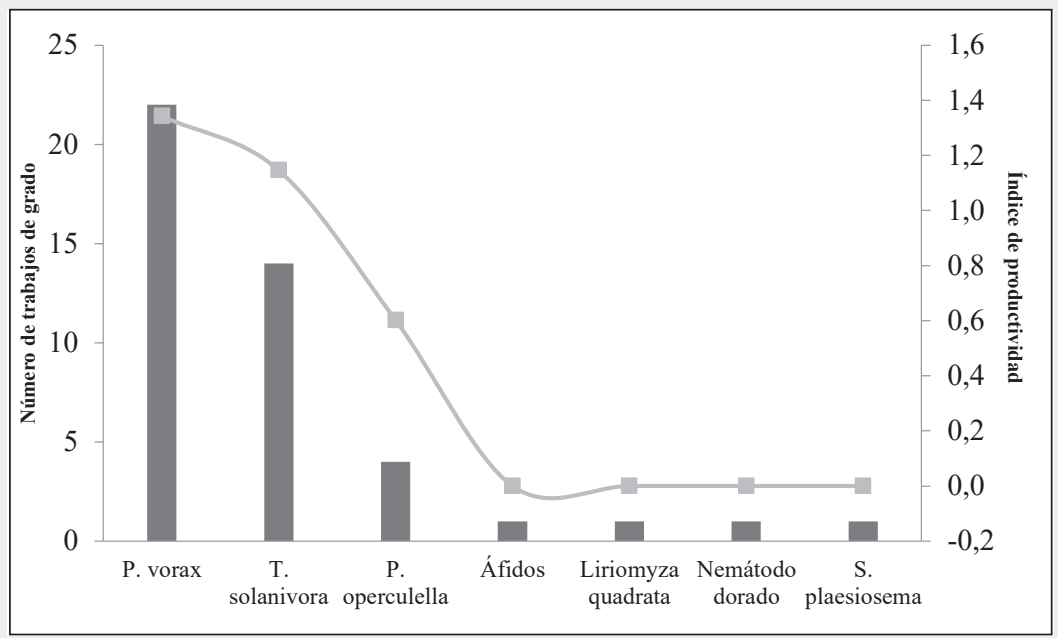

Gráfica 5. Áreas específicas e índices de productividad incluidas en trabajos de grado para el cultivo de papa en la línea de entomología, durante los años 1967 a 2012. Escuela Ingeniería Agronómica, UPTC, Tunja-Boyacá, Colombia.

\subsection{Nutrición}

Durante los años 1989a 1999 se documentaron 19 trabajos de grado equivalentes al 54,2\%, seguido del periodo 1978-1988 con 11, correspondiente al 31,4\%. Para 2000 a 2012 fueron reportados 4 trabajos $(11,4 \%)$; evidenciándose con un único estudio el menor número de investigaciones (2,85\%) para los años 1967 a 1977. 


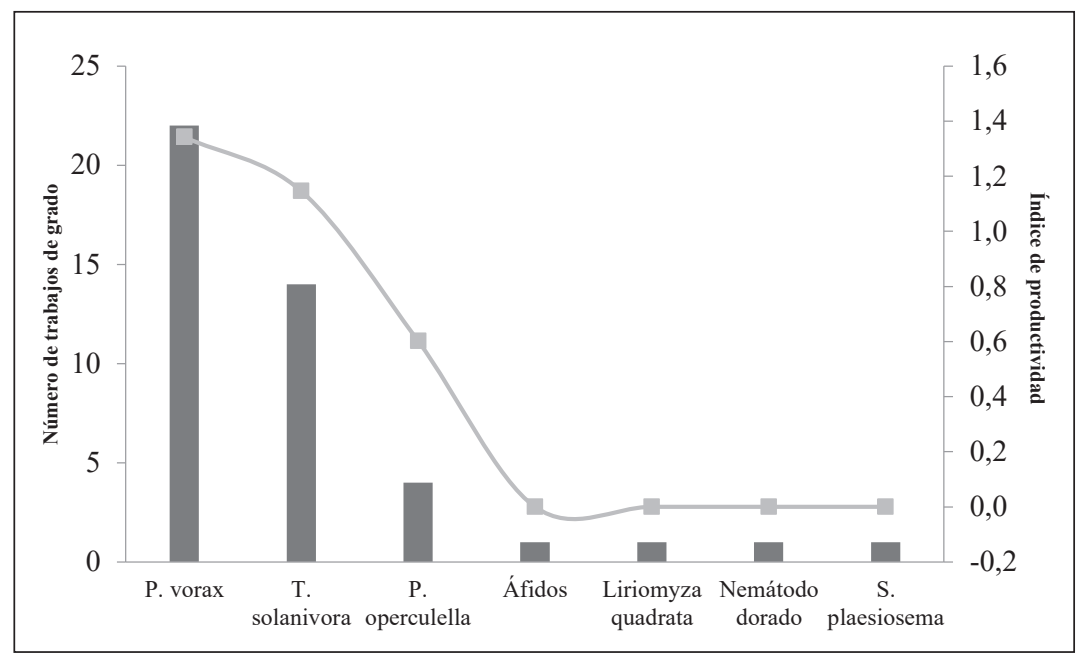

Gráfica 6. Áreas específicas e índices de productividad incluidas en trabajos de grado para el cultivo de papa en la línea de Nutrición, durante los años 1967 a 2012. Escuela Ingeniería Agronómica, UPTC, Tunja-Boyacá, Colombia.

Los trabajos de grado más estudiados, correspondieron a la nutrición edáfica con N-P-K $(27,02 \%)$ y fósforo $(24,3 \%)$; mientras que la potásica $(5,4 \%)$ y los potencializadores de la fertilización $(2,5 \%)$ fueron los menos investigados (Gráfica 6). En los programas de fertilización química cobra importancia el uso de enmiendas al mejorar las propiedades fisicoquímicas del suelo, además de aumentar rendimientos en producción [17]. Según [18], se optimizan las propiedades físicas y biológicas de los suelos arcillosos usando sulfato ferroso. De acuerdo con [19], [20], [21], existe aumento en producción al emplear la fertilización edáfica N-P-K, fósforo $\mathrm{y}$ abonos orgánicos como compost, gallinaza y bovinaza [22], y la incorporación de enmiendas para el manejo de la acidez en el suelo [23], [24]. En esta área específica se evaluó un considerable número de trabajos, debido a la importancia de los macronutrientes en el desarrollo del cultivo [25], [26], [27]. Para suplir las deficiencias de fósforo en el cultivo se recomienda el uso del superfosfato triple [28] y de los fertilizantes compuestos [29], [30]. Al respecto [31], encontró sobre el "Cv Diacol capiro" efecto de respuesta a la fertilización en un suelo andisol con 3 niveles de $\mathrm{N}, \mathrm{P}, \mathrm{K}$ en mezcla con $\mathrm{Ca}, \mathrm{Mg}$ y B.

\subsection{Fisiología Vegetal}

Durante el periodo 2000 a 2012 se adelantaron 14 trabajos de grado, correspondientes al $70 \%$, seguido por el de los años 1967-1977 con 3 (15\%). Para 1978 a 1988 se registraron 2 estudios (10\%); evidenciándose el desarrollo del menor número de trabajos de grado con una unidad (5\%) para los años 1989 a 1999.

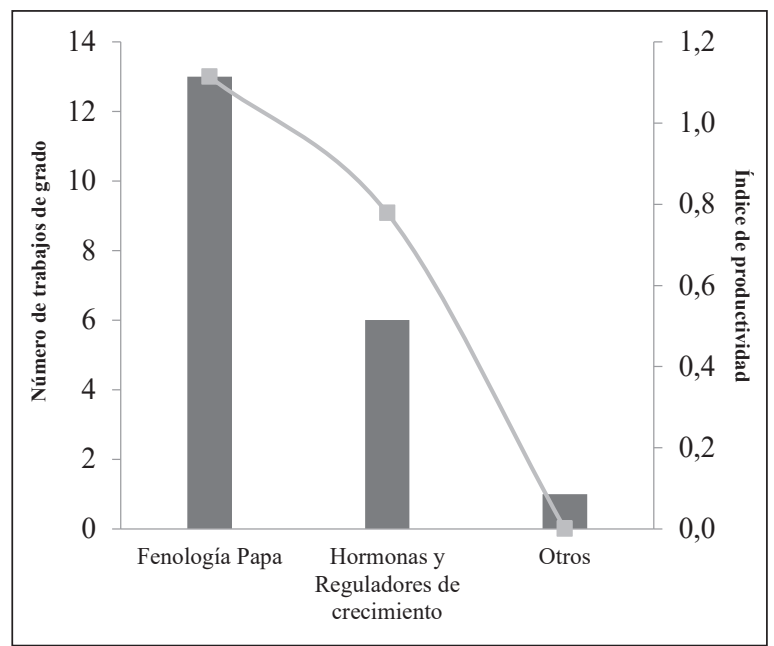

Gráfica 7. Áreas específicas e índices de productividad evaluadas en trabajos de grado para el cultivo de papa en la línea de fisiología vegetal durante los años 1967 a 2012. Escuela Ingeniería Agronómica, UPTC, Tunja-Boyacá, Colombia.

La mayoría de trabajos en fisiología vegetal $(65 \%)$, se direccionaron a la investigación de 
estados fenológicos, crecimiento y desarrollo del cultivo de papa (Gráfica 7). De acuerdo con [32], el crecimiento se define como el incremento de tamaño, representado en el aumento de volumen, peso, número de células, cantidad de protoplasma y complejidad. En los trabajos de grado analizados, el crecimiento y desarrollo del cultivar Diacol Capiro reflejó el mayor ciclo vegetativo con 135 días después de emergencia (dde) [33], contrario a lo expuesto por [34] con 130 dde y [35] 120 dde. Para el caso de la variedad Parda Pastusa [36] y [37], encontraron que el desarrollo y crecimiento requiere de 180 dde, lo cual difiere de [38] con 150 dde y [35] 135 dde, siendo este el menor tiempo de desarrollo. En S. Phureja var Criolla Colombia se encontró que el desarrollo y crecimiento se da a los 120 dde [35]; por su parte [39], reporta 115 dde y [35] un menor tiempo con 105 dde; en general se necesitan 105 a 120 dde. En comparación a las anteriores variedades, la papa criolla presenta el menor tiempo para su crecimiento y desarrollo. [35], al evaluar en Diacol capiro, parda pastusa y criolla, observó un menor tiempo en los días de emergencia para los tres materiales frente a lo encontrado por los demás autores que estudiaron esta variable, siendo $S$. tuberosum phureja la más precoz (105 dde).

Según [40], son importantes los análisis de crecimiento y relación fuente-demanda de los materiales de interés comercial, para obtener los índices de crecimiento y los potenciales de fuente en aras de mejorar la producción.

\subsection{Economía}

Se entiende por evaluación agroeconómica como la ciencia que explica el concepto, objeto, métodos y algunas particularidades de la producción agropecuaria y su desarrollo socioeconómico [37]. El mayor número de estudios relacionados con esta área se encontró para los años 1978-1988 con 10 proyectos $(55,6 \%)$. Para el periodo 2000 a 2012 se realizaron 5 trabajos $(27,8 \%)$, reduciendo el número de investigaciones durante los años 1989 a 1999 y 1967 a 1977 a $2(11,1 \%)$ y 1 $(5,6 \%)$, respectivamente.

Tabla II. Áreas específicas desarrolladas en trabajos de grado para la línea economía. Escuela de Ingeniería Agronómica, UPTC.

\begin{tabular}{|c|l|c|c|}
\hline No. & \multicolumn{1}{|c|}{ TEMA } & No. ESTUDIOS & PORCENTAJE (\%) \\
\hline 1 & Evaluación y respuesta agroeconómica en fertilización & 10 & 50 \\
\hline 2 & Producción y mercadeo & 3 & 15 \\
\hline 3 & Evaluación económica en variedades de papa & 2 & 10 \\
\hline 4 & Respuesta agroeconómica en sistemas de siembra & 1 & 5 \\
\hline 5 & Análisis de la demanda & 1 & 5 \\
\hline 6 & Evaluación económica de un insecticida & 1 & 5 \\
\hline 7 & Valor económico y nutritivo de papa rich0065. & 1 & 5 \\
\hline 8 & Análisis de comercialización en función de almacenamiento. & 1 & 5 \\
\hline
\end{tabular}

En los trabajos de grado adelantados, se destaca la evaluación agroeconómica de fertilizantes con 10 estudios $(50 \%)$ (Tabla II), ensayando dosis, mezclas, formas de aplicación y fuentes de fertilización buscando la mejor relación costo-beneficio. Se pone de relieve la nutrición fosfórica, ya que esta favorece un mejor desarrollo de raíces, crecimiento aéreo, aceleración de la maduración e intermediación en la formación de azúcares y almidón [41]. En el cultivo de papa el fosforo interviene en el número y tamaño de tubérculos, el cual es absorbido con dificultad por la planta debido a su sistema radical y baja movilidad hacia la raíz [42]. El cloruro de potasio se reporta como la mejor fuente para aumentar rendimientos e ingresos [43], lo cual difiere de autores como [44], quienes indican que las fuentes a base de fósforo son más rentables y afirman que para obtener utilidades hasta del $28,5 \%$ se deben usar los fertilizantes grado 14-14-14 más roca fosfórica, mientras [45] argumentan que una rentabilidad de $175,5 \%$ se obtiene al aplicar fósforo hidrosoluble y citrosoluble en mezcla, lo cual es corroborado por [46] quienes mencionan que un mayor ingreso se consigue 
No. 2

Julio - Dic. 2017

ISSN 0122-820X

E-ISSN 2422-5053

PP: $102-115$ mezclando $25 \%$ de fósforo hidrosoluble + $75 \%$ de fósforo citrosoluble + materia orgánica + nitrógeno y potasio. Según [47], la papa tiene una participación de los fertilizantes en el costo total de producción de un $17-20 \%$. De acuerdo con [48], la fertilización con abono químico 13-26-6 y orgánico en dosis de $300-800 \mathrm{~kg} \mathrm{ha}^{-1}$ promueven los mayores rendimientos de $S$. phureja, con una relación costo beneficio (C/B) de 1,47.

\subsection{Fitopatología}

Durante los años 1978 a 1988 y 2000 a 2012 se desarrolló el mayor número de trabajos de grado, con 5 investigaciones $(33,3 \%)$ en cada lapso de tiempo. Para 1989 a 1999 se hicieron 3 trabajos (20\%), seguido por los años 19671977 con 2 estudios $(13,3 \%)$.

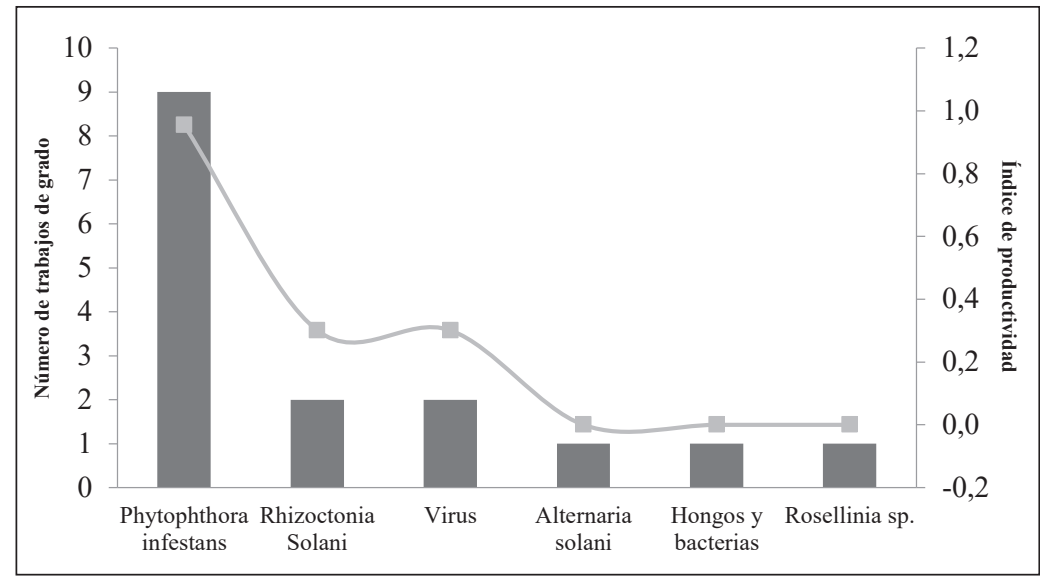

Gráfica 8. Áreas específicas e índices de productividad, desarrolladas en trabajos de grado para el cultivo de papa en la línea de fitopatología durante los años 1967 a 2012. Escuela Ingeniería Agronómica, UPTC, Tunja-Boyacá, Colombia.

El control químico para Phytophthora infestans (56,3\%) (Gráfica 8), toma importancia en Colombia desde 1876 cuando se reportó por primera vez este patógeno, causando daños entre $8 \%$ y $12 \%$ de los costos de producción total del cultivo [49]. La enfermedad destruye el follaje de la planta y tallos llegando hasta el tubérculo. Al principio se forman por lo general en las puntas o bordes de las hojas inferiores manchas circulares irregulares acompañadas de una zona blanca en el envés, correspondiente a las hifas [50]. Para el control químico se reporta con éxito el uso del ingrediente activo (i.a) Mancozeb [51] empleando dosis de $3 \mathrm{~kg} \mathrm{ha}^{-1}$, [52] 0,75 $\mathrm{Kg} \mathrm{ha}^{-1} \mathrm{y}$ [53] $4 \mathrm{Kg} \mathrm{ha}^{-1}$. Los cultivares Parda pastusa y Tuquerreña fueron evaluados por presentar moderada y alta susceptibilidad al patógeno, respectivamente [54]. Resultados similares fueron corroborados por [55], quienes mostraron eficiencia del fungicida Previcur ${ }^{\circledR}$ al reducir de manera significativa la incidencia de la enfermedad, comparado con el fungicida Mancozeb de uso común por productor.

\section{Conclusiones}

Existen 1098 trabajos de grado, realizados durante 55 años de trayectoria del programa de Ingeniería Agronómica de la Universidad Pedagógica y Tecnológica de Colombia, de los cuales 130 corresponden a las cinco áreas seleccionadas para la revisión. En relación con el área de entomología (42 tesis, 32,3\%), los trabajos sobre gusano blanco (P. vorax) fueron los más representativos. En fertilización (35 tesis, 26,9\%), las áreas específicas a resaltar corresponden a nutrición con elementos mayores N-P-K, haciendo énfasis en la 
Lizeth Johana Sierra-Ariza, Katherin Dayana Suárez-Roberto, Jorge Enrique Villamil-Carvajal, Álvaro Enrique Alvarado-Gaona

evaluación del uso del fósforo. En fisiología vegetal (20 tesis, 15,4\%), se documentaron en mayor proporción estudios sobre estadios fenológicos, crecimiento y desarrollo del cultivo de papa. En economía (18 tesis 13,8\%), fue más importante la evaluación económica de la respuesta agronómica en fertilización, y finalmente en fitopatología (15 tesis, 11,5\%), el tizón tardío ( $P$. infestans) correspondió al patógeno más estudiado.

Esta revisión brinda información a estudiantes, profesionales e instituciones involucradas con el sector papero colombiano y departamental, para la orientación y enfoque de futuros trabajos de investigación.

\section{Referencias}

[1] R. Martin y R. Jerez, "Evaluación del rendimientoenpapa(Solanumtuberosum, L.) a partir del comportamiento de las temperaturas", Cultivos Tropicales, vol. 36, no. 1, pp. 93-97, 2015.

[2] Manual Papa. Cámara de Comercio de Bogotá y Núcleo Ambiental S.A., Bogotá, 2015.

[3] N. Borba, "La papa un alimento básico, Posibles impactos frente a la introducción de papa transgénica", RAP-AL en Uruguay, 2008. [En línea]. Disponible en: www.rapaluruguay.org/ transgenicos/Papa/Papa.pdf [Accedido: 2-may-2016]

[4] O. Villafuerte, "La papa o patata, año internacional de la papa", Agroancash, Colombia, 2008. [En línea]. Disponible en: http://www.agroancash.gob. pe/public/articulos/aip2008/temas/ botanica.html [Accedido: 18-jun-2016]

[5] P. Porras y Y. López, “Caracterización del proceso productivo de la papa en Colombia con énfasis en ecosistemas de páramo", en Guía ambiental para el cultivo de la papa, Bogotá D.C, Colombia: Diagráficas Ministerio de Agricultura y Desarrollo RuralFEDEPAPA. 2004.

[6] M. Vallejo, "Estudio longitudinal de la producción Española de tesis doctorales en Educación Matemática (1975-2002)", tesis doctoral, Universidad de Granada, España, 2005.

[7] S. Arenas y A. Romero, "Indicadores bibliométricos de la revista científica Zootecnia Tropical", Zootecnia Tropical, vol. 21, no. 3, pp. 325-350, 2003.

[8] D.S. Price, "A general theory of bibliometric and other cumulative advantage processes", Journal of the American Society for Information Science, vol. 27, no. 5, pp. 292-306, 1976.

[9] S.A. Gutiérrez Umaña y I.M. Pereira Suárez, "Evaluación del insecticida isazofos en el manejo y control del gusano blanco de la papa Premnotrypes vorax (Hustache) en el municipio de Motavita-Boyacá", Universidad Pedagógica y Tecnológica de Colombia, Boyaca, Colombia, 1991.

[10] L. Torres, P. Gallegos, C. Castillo y C. Asaquibay, "Manejo de Gusano Blanco", International Potato Center, 2013.

[11] A.E. Alvarado, "80 años del Gusano Blanco de la Papa en Colombia", Red de papa, vol.1, no. 1, pp. 12-113, 2002.

[12] O. Camacho y S. Fajardo, "Evaluación de insecticidas en el control del gusano blanco de la papa (Premnotrypes vorax Hustache)", trabajo de fin de grado, Universidad Pedagógica y Tecnológica de Colombia, 1975.

13] A. Moreno y D. Charria, "Producción y mercadeo de papa en el área central de Boyacá", Ingeniería Agronómica. Facultad Ciencias Agropecuarias. UPTC. Tunja, Colombia, 1980. 
Vol. 22

No. 2

Julio - Dic. 2017

ISSN 0122-820X

E-ISSN 2422-5053

PP: 102-115
[14] H. Calvache, "Efectos de barreras vegetales y químicas en el control del gusano blanco de la papa", Revista Latinoamericana de la papa, vol. 1, no. 1, pp. 22, 1991.

[15] P. Larral, J. Montenegro y R. Ripa, "Control químico", En: Manejo de plagas en paltos y cítricos, Primera edición. Chile; INIA, 2008.

[16] E.E. Ebrat y C.A. Madero, "Evaluación de hongos entomopatógenos para el control de gusano blanco de la papa Premnotrypes vorax (Hustache) en el municipio de Motavita-Boyacá", Ingeniería Agronómica. Facultad Ciencias Agropecuarias. UPTC. Tunja, Colombia, 1995.

[17] G. Arévalo y M. Castellano, "Manual Fertilizantes y Enmiendas", Zamorano, Honduras; PROMIPAC, 2009.

[18] A.B. Castro y B.R. Valbuena, "Evaluación de un suelo arcilloso mejorado con sulfato ferroso con base en un segundo cultivo papa Solanum phureja", Ingeniería Agronómica. Facultad Ciencias Agropecuarias. UPTC. Tunja, Colombia, 1992.

[19] F. Forero "Mezcla física de abonos, una alternativa en la mediana y pequeña empresa agrícola", Colombia; Uniediciones Ltda, 2010..

[20] H. Grijalba, "Respuesta de la papa Solanum tuberosum L. a la fertilización edáfica con abono orgánico y micorrizas en Siachoque Boyacá", Ingeniería Agronómica. Facultad Ciencias Agropecuarias. UPTC. Tunja, Colombia, 1992.

[21] N.A. Sánchez, "Respuesta del cultivo de papa (Solanum tuberosum L.) a la fertilización con una fuente química y dos orgánicas en el municipio Cómbita Boyacá”, Ingeniería Agronómica.
Facultad Ciencias Agropecuarias. UPTC Tunja, Colombia, 2012.

[22] W. Garcés y M. Salamanca, "Respuesta agroeconómica del cultivo de la papa Solanum tuberosum L. a la fertilización con abono orgánico reforzado", Ingeniería Agronómica. Facultad Ciencias Agropecuarias. UPTC. Tunja, Colombia, 1993.

[23] I. Neira y J.O. Panqueva, "Aplicación de tres fuentes de materia orgánica como complemento a la fertilización con 13-26-6 en papa criolla yema de huevo (Solanum phureja) Jez et buk en Toca Boyacá", Ingeniería Agronómica. Facultad Ciencias Agropecuarias. UPTC. Tunja, Colombia, 1996.

[24] A.A Uribe y I. Moncada, "Comparación de dos métodos de encalada en el cultivo de papa". Ingeniería Agronómica. Facultad Ciencias Agropecuarias. UPTC. Tunja, Colombia, 1978.

[25] C.R Restrepo y O. Auzaque, "Efecto de correctivos y dosis en suelos ácidos para la producción de papa Solanum tuberosum L. en Soracá Boyacá", Ingeniería Agronómica. Facultad Ciencias Agropecuarias. UPTC. Tunja, Colombia, 1993.

[26] J. Porta, M. Acevedo y R. Poch, "Edafología uso y protección de suelos", 3 ed. Madrid, España; Mundi-Prensa, 2014.

[27] J. Castro y E. García, "Influencia del fósforo hidro y citrosoluble de los abonos fosfóricos en la producción de papa", Ingeniería Agronómica. Facultad Ciencias Agropecuarias. UPTC. Tunja, Colombia, 1979.

[28] M. Rodríguezy M. Villabona, "Influencia del fósforo hidrosoluble e insoluble en la producción del cultivo de papa (Solanum tuberosum)", Ingeniería Agronómica. 
Facultad Ciencias Agropecuarias. UPTC. Tunja, Colombia, 1982.

[29] A. Gaitán, "Producción de papa (Solanum tuberosum L.) utilizando métodos de fertilización por inmersión de tubérculos en soluciones de fósforo", Ingeniería Agronómica. Facultad Ciencias Agropecuarias. UPTC. Tunja, Colombia, 1983.

[30] S.C. Orjuela y C.E. Vega "Efecto de la micorriza-vesiculo arbuscular sobre la eficiencia de la fertilización fosfórica en papa criolla amarilla variedad "yema de huevo" (Solanum phureja Juz et Buk) en suelos del municipio de Motavita, Boyacá”, Ingeniería Agronómica. Facultad Ciencias Agropecuarias. UPTC. Tunja, Colombia, 1995.

[31] E.D. Tabares, S. Jaramillo, L. H. González, J. M. Cotes, "Respuesta de la papa (Solanum tuberosum L.) variedad DIACOL CAPIRO a la fertilización en un Andisol del Oriente Antioqueño, Colombia", Revista Facultad Nacional de Agronomía Medellín, vol. 62, no. 2, pp. 5059-5110, 2009.

[32] N.A. Cuchimaque y M. Sierra, "Evaluación de materiales fosfóricos parcialmente acidulados como complemento a la fertilización tradicional en el cultivo de la papa (Solanum tuberosum var. ICA- Morita), en Ventaquemada (Boyacá)", Ingeniería Agronómica. Facultad Ciencias Agropecuarias. UPTC. Tunja, Colombia, 1998.

[33] F. Salisbury y C. Ross, Fisiología de la planta 3, desarrollo de la planta y fisiología ambiental, Madrid, España; COPYRIGHT International Thomson editores Spain paraninfo S.A, 2000.

[34] E.D. Acero, "Reconocimiento de los estadios fenológicos en función de los grados día de crecimiento en papa

(Solanum Tuberosum L) variedad Diacol Capiro, en Ventaquemada - Boyacá", Ingeniería Agronómica. Facultad Ciencias Agropecuarias. UPTC. Tunja, Colombia, 2009.

[35] C.E. Pedraza, "Análisis de los estadios fenológicos, crecimiento y desarrollo en papa (Solanum tuberosum L.) variedad Diacol Capiro (subespecie andigena), en Ventaquemada - Boyacá.)", Ingeniería Agronómica. Facultad Ciencias Agropecuarias. UPTC. Tunja, Colombia, 2008.

[36] M.N. Reyes, "Caracterización fenológica de tres variedades de papa en tiempo fisiológico bajo condiciones climáticas de Ventaquemada Boyacá", Ingeniería Agronómica. Facultad Ciencias Agropecuarias. UPTC. Tunja, Colombia, 2010.

[37] G.E. Martínez, “Análisis de los estadios fenológicos, crecimiento y desarrollo en papa (Solanum tuberosum L.) variedad parda pastusa (subespecie andígena), en Ventaquemada - Boyacá", Ingeniería Agronómica. Facultad Ciencias Agropecuarias. UPTC. Tunja, Colombia, 2008.

[38] J.R. Niño, "Análisis del crecimiento y desarrollo en el cultivo de papa (Solanum tuberosum L.) variedad parda pastusa en condiciones del municipio de Ventaquemada Boyacá", Ingeniería Agronómica. Facultad Ciencias Agropecuarias. UPTC. Tunja, Colombia, 2010.

[39] F. Mesa, "Crecimiento y desarrollo de papa (Solanum tuberosum variedad parda pastusa) en el municipio de Ventaquemada Boyacá", Ingeniería Agronómica. Facultad Ciencias Agropecuarias. UPTC. Tunja, Colombia, 2009. ol. 22 No. 2 2017 .

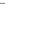


No. 2
"Análisis de crecimiento y relación fuente-demanda de cuatro variedades de papa (Solanum tuberosum L.) en el municipio de Zipaquirá (Cundinamarca, Colombia)", Revista Facultad Nacional de Agronomía Medellín, vol. 63, no. 1, pp. 5253-5266, 2010.

[41] C.C. Higuera, "Comportamiento de los estadios fenológicos en función de los grados día de crecimiento en Solanum phureja en el municipio de Ventaquemada, Boyacá", Ingeniería Agronómica. Facultad Ciencias Agropecuarias. UPTC. Tunja, Colombia, 2009.

[42] J.M. Pardo, "Determinación del crecimiento y estadios fenológico de Solanum phureja (Juz.et Buk): var. Criolla Colombia en el municipio de Ventaquemada-Boyacá.)", Ingeniería Agronómica. Facultad Ciencias Agropecuarias. UPTC. Tunja, Colombia, 2008.

[43] E. Vivas, Universidad Nacional Agraria, Apoyo financiero agencia sueca para el desarrollo internacional (Asdi) en Nicaragua, "Economía agraria", 2010. [En línea] Disponible en: repositorio. una.edu.ni/2465/1/ne10v855e.pdf. [Accedido: 19-dic-2016]

[44] H. Castro, Fundamentos para el conocimiento y manejo de suelos agrícolas, Tunja, Colombia; Produmedios, Instituto Universitario Juan de Castellanos, 1998.

[45] C. Herrera, L. Fierro y J. Moreno, Manejo integrado del cultivo de papa manual técnico, Bogotá, Colombia; Produmedios, Corpoica, Ministerio de agricultura y desarrollo rural, 2000.

[46] E.G. Chinchilla y L. F. Santamaria, "Respuesta agroeconómica de la papa (Solanum tuberosum) a dos fuentes de potasio en el municipio de Motavita",
Ingeniería Agronómica. Facultad Ciencias Agropecuarias. UPTC. Tunja, Colombia, 1978.

[47] O. Gómez y O. Granados, "Respuesta agroeconómica de la papa (Solanum tuberosum ssp. andigena) a sistemas de siembra y dosis de fertilización en Samacá (Boyacá)", Ingeniería Agronómica. Facultad Ciencias Agropecuarias. UPTC. Tunja, Colombia, 1982.

[48] L. Muñoz, A. Lucero, "Efecto de la fertilización orgánica en el cultivo de papa criolla Solanum phureja", Agronomía Colombiana, vol. 26, no 2, pp. 340-346, 2008.

[49] C. Prieto y C. A. Serrano, "Respuesta agroeconómica del cultivo de la papa (Solanum tuberosum L.) a la aplicación de fosforo hidrosoluble y citrosoluble en un suelo fuertemente acido", Ingeniería Agronómica. Facultad Ciencias Agropecuarias. UPTC. Tunja, Colombia, 1983.

[50] E.F. Arias y F. H. Gonzales, "Respuesta agroeconómica del cultivo de la papa (Solanum tuberosum L.) en suelos ácidos a dos fuentes de fosforo en diferentes proporciones en el municipio de TutaBoyacá", Ingeniería Agronómica. Facultad Ciencias Agropecuarias. UPTC. Tunja, Colombia, 1988.

[51] J. Pérez, "Uso de los fertilizantes y su impacto en la producción agrícola", trabajo de grado M.S, DepartamentoBiociencias, Facultad de Ciencia. Universidad Nacional de Colombia. Tunja, Colombia, 2014.

[52] N. Carreño et al., "Problemas fitopatológicos en especies de la familia Solanácea causados por los géneros Phytophthora, Alternaría y Ralstonia en Colombia - Una revisión", Agronomía Colombiana, vol. 25, no. 2, pp. 321, 2007 
[53] G. Agrios, Fitopatología, 2nd ed. México; Limusa, 1996. pp. 303-322.

[54] D.F. Álvarez y J. F. Plazas, "Control químico de la gota de la papa Phytophthora infestans (mont) de bary en la zona de tierra negra (Boyacá)", Ingeniería Agronómica. Facultad Ciencias Agropecuarias. UPTC. Tunja, Colombia, 1986.

[55] H.G. García, M. Marín, S. Jaramillo, J.M. Cotes, "Sensibilidad de aislamientos colombianos de Phytophthora infestans a cuatro fungicidas sistémicos", Agronomía colombiana, vol. 26, no. 1, pp. 47-57, 2008. 